\title{
The Synergetic and Multifaceted Nature of Carbon-Carbon Rotation Reveals the Origin of Stability for Bulky Alkane Dimers
}

\author{
Xinjie Wan, ${ }^{1}$ Xin He, ${ }^{1}$ Meng Li, ${ }^{1}$ Bin Wang,,${ }^{1,2}$ \\ Chunying Rong, ${ }^{1 *}$ and Shubin Liu ${ }^{3,4^{*}}$ \\ ${ }^{1}$ Key Laboratory of Chemical Biology and Traditional Chinese Medicine Research \\ (Ministry of Education of China), Hunan Normal University, \\ Changsha, Hunan 410081, P.R. China \\ E-mail: rongchunying@aliyun.com \\ ${ }^{2}$ Eenheid Algemene Chemie, Vrije Universiteit Brussel, Faculteit Wetenschappen, Pleinlaan 2, \\ 1050 Brussels, Belgium \\ ${ }^{4}$ Research Computing Center, University of North Carolina, Chapel Hill, North Carolina 27599- \\ 3420, USA. E-mail: shubin@email.unc.edu \\ ${ }^{5}$ Department of Chemistry, University of North Carolina, Chapel Hill, North Carolina, 27599- \\ 3290, USA
}

\begin{abstract}
Designing compounds with as long carbon-carbon bond distances as possible to challenge conventional chemical wisdom is of current interest in the literature. These compounds with exceedingly long bond lengths are commonly believed to be stabilized by dispersion interactions. In this work, we build nine dimeric models with varying sizes of alkyl groups, let the carbon-carbon bond flexibly rotate, and then analyze rotation barriers with energy decomposition and information-theoretic approaches in density functional theory. Our results show that these rotations lead to extraordinarily elongated carbon-carbon bond distances and rotation barriers are synergetic and multifaceted in nature. The dominant factor contributing to the stability of the dimers with bulky alkane groups is not the dispersion force but the electrostatic interaction with steric and exchange-correlation effects playing minor yet indispensable roles.
\end{abstract}


The covalent bonding between carbon atoms is among the most fundamental and extremely important concept in chemistry from the perspective of structure, stability, and reactivity. One of the recent endeavors pertaining to this perspective is to synthesize compounds with $\mathrm{C}-\mathrm{C}$ single covalent bond distance as long as possible. While a typical $\mathrm{C}-\mathrm{C}$ bond length is $1.54 \AA$, reports of experimentally synthesized compounds with a bond distance longer than $1.8 \AA$ or even $2.0 \AA$ are available in the literature. ${ }^{1-6}$ These spectacular accomplishments sparked a cohort of investigators looking into the mechanism of their underlying interactions. ${ }^{1,7-9}$ It is commonly believed that weak, van der Waals interactions, especially dispersion forces, are responsible for the formation and stability of these exceedingly long carbon-carbon single bonds. However, there are lingering questions about these species. How can the stability of a covalent bond be dominated by noncovalent interactions? More importantly, in quantum mechanics, there is no specific term in the Hamiltonian associated with van der Waals interactions. How can these weak interactions be reflected by energetics or other electronic properties?

In this computational study, to address these questions, we design a series of bulky alkane dimers with the varying size of terminal groups and thus different $\mathrm{C}-\mathrm{C}$ distances between the two bulky groups (Scheme 1), and then let the $\mathrm{C}-\mathrm{C}$ bond of these species to rotate 360 degrees (see Computational Details in $S /$ ). Patterns of the results emerged from these processes and analyses thereafter should provide us with clues about the origin of their stability. To analyze the results, we employ two schemes of the density-based total energy decomposition, ${ }^{10-14}$ information-theoretic approach (ITA), ${ }^{15-18}$ and natural bond orbital (NBO) ${ }^{19}$ analyses, whose details are available elsewhere. ${ }^{20}$

Figure 1 illustrates the profile of the $\mathrm{C}$ - $\mathrm{C}$ distance as a function of its flexible rotation from $0^{\circ}$ to $360^{\circ}$ with the step size of $5^{\circ}$. From the Figure, we can see that for some systems such as 1-3, the $\mathrm{C}-\mathrm{C}$ distance does not change much during the rotation process, whereas for others such as $\mathbf{5 - 8}$, marked elongation of the $\mathrm{C}-\mathrm{C}$ bond is discernible. For example, for $\mathbf{6}$, its optimal 
distance in the equilibrium is $1.78 \AA$, but it becomes $2.00 \AA$ when the two bulky groups are rotated $220^{\circ}$. Another example is $\mathbf{9}$, whose shortest C-C distance is $1.688 \AA$ but the longest value is $1.820 \AA$. Figure 2 shows the corresponding energetic profile for the above process for each of the nine compounds. Smooth curves are seen for $\mathbf{1}$ and $\mathbf{4}$, indicating that the two bulky alkane groups of these two compounds can be freely rotated. For the remaining systems, the curves are not smooth, suggesting that there are steric clashes between the two bulky groups during the rotation process. When a steric clash happens, significant structural and energetic changes are resulted, leading to the discontinuity of the energy profile. Figure 2 also shows that since each of these species was uniquely built, so none of these energetic profiles is the same as others. The profiles of $1 \& 4$, and $\mathbf{7} \& \mathbf{8}$ look similar, but when looking into details, we find that they are indeed different.

Table 1 shows the correlation coefficient $(\mathrm{R})$ result of the total energy difference $\Delta \mathrm{E}$ for each of the nine systems with numerous quantities from the total energy decomposition analysis, information-theoretic approach, and NBO analysis. A positive sign of the correlation coefficient indicates that $\Delta \mathrm{E}$ is positively correlated with the given quantity. A negative value means otherwise. From the Table, we can see that some quantities are strongly and uniformly correlated with all nine systems. Examples include five energetic components, ${ }^{10}$ Fisher information $^{21} \Delta \mathrm{l}_{\mathrm{F}}$, Ghosh-Berkowitz-Parr entropy ${ }^{22} \Delta \mathrm{S}_{\mathrm{GBP}}$ and the alternative relative Fisher information $\Delta \mathrm{G}_{1} \cdot{ }^{23,24}$ For other quantities tabulated in the Table, strong correlations can be found for one or few systems, but not for all nine. For instance, for the total hyperconjugation interactions, ${ }^{19} \Delta \mathrm{E}$ is seen to be strongly and negatively correlated with it in Compounds $\mathbf{1 , 2}$ and 4, suggesting that the effect of hyperconjugation can be utilized to quantitatively described the conformational stability for these systems. The lower the total energy, the stronger the hyperconjugation effect, suggesting that effects from non-Lewis bonding interactions can be important for these species. However, for others, this effect is no longer valid, and could even qualitatively untrue, as shown for Compounds $\mathbf{6}$ and $\mathbf{7}$ with a positive 
correlation coefficient value. These results in Table1 are at the molecular level.

Zooming in to the two carbon atoms of the $\mathrm{C}-\mathrm{C}$ bond, we also obtained strong correlation results from the above analyses, as exhibited in Table 2, where we considered the $\mathrm{C}-\mathrm{C}$ bond distance, Hirshfeld charge,${ }^{25,26}$ local temperature,${ }^{27}$ and numerous atomic ITA quantities using the Hirshfeld partition for the carbon atoms of the C-C bond.$^{20}$ As can be seen from the Table, like the trends from Table 1, some quantities such as local temperature, Fisher information, and relative Fisher information are strongly correlated with $\Delta E$ for all species, whereas others are only partly.

These results allude us to at least two viewpoints. First, each system is special with its own characteristics, as demonstrated by the unique C-C distance profile in Fig. 1, distinct energetics profile in Fig. 2, and different behaviors of correlation tendencies in Tables 1 and 2. This is apparently due to the different size of the bulky alkane groups, leading to varying molecular structures and properties. Secondly, these results also unveil that there are common patterns arising from the correlation analyses. For some properties, the nine systems all yielded the same strong correlation. Examples at the molecular level include energy components such as $\Delta \mathrm{E}_{\mathrm{xc}}, \Delta \mathrm{E}_{\mathrm{e}}, \Delta \mathrm{E}_{\mathrm{s}}, \Delta \mathrm{T}_{\mathrm{S}}$ and information-theoretic quantities like $\Delta \mathrm{I}_{\mathrm{F}}, \Delta \mathrm{G}_{1}$, and $\Delta \mathrm{S}_{\mathrm{GBP}}$, where $\Delta \mathrm{E}$ has been found to strongly correlates with them for all nine compounds. At the atomic level, from Table 2, we see that local temperature at both carbon atoms of the $\mathrm{C}-\mathrm{C}$ bond, and atomic information-theoretic quantities such as $\Delta \mathrm{I}_{\mathrm{F}}, \Delta \mathrm{G}_{1}$, and $\Delta \mathrm{G}_{3}$ consistently generate strong correlations across all nine systems.

The fact that $\Delta \mathrm{E}$ strongly correlates with several electronic properties simultaneously for all the systems studied in this work firmly suggests that the energy barrier generated by the C-C single bond flexible rotation was resulted from the effects that are synergetic and multifaceted in nature. Since different electronic properties have distinctly different physical meanings, ${ }^{14,15,20}$ these multiple strong correlations elucidate that many different effects cooperatively come into play during the $\mathrm{C}-\mathrm{C}$ rotation process. For example, a strong 
correlation of $\Delta \mathrm{E}$ with $\Delta \mathrm{E}_{\mathrm{e}}$ suggests that the electrostatic interaction is a reliable descriptor of the rotation barrier. ${ }^{11}$ On the other hand, $\Delta \mathrm{E}$ is also strongly correlated with $\Delta \mathrm{E}_{\mathrm{s}}$ and $\Delta \mathrm{E}_{\mathrm{xc}}$ components, illustrating that the steric effect and quantum effect from exchange-correlation interactions are also quantitative descriptors. This is also true for the case of a few ITA quantities. These superficially contradicting results signify the need to appreciate the origin of the rotation barrier in a systems manner, where its nature is not just dictated by one factor but, instead, concurrently by many ones.

Back to our original question about the role of van der Waals interactions, which was believed in the literature ${ }^{1,5-9}$ to be mainly responsible for the stability of these dimers with bulky alkane groups, our results from noncovalent interaction analyses as quantified by nonLewis orbital contributions, C-C bond occupancy, and hyperconjugation effect show that for some systems they are strongly correlated, but for others they are not the case. This observation is supported by the benchmark results shown in Table 3 for Compound $\mathbf{5}$, where little dependence of our results on the choice of basis sets and methods is clearly demonstrated. In particular, as shown from the Table, approximate density functionals with and without the inclusion of the dispersion correction ${ }^{28}$ do not really make any difference. These results raise the question of whether dispersion forces are the real dominant interaction putting the two bulky alkane groups together.

To further understand the stability origin of these bulky alkane dimers, we combine all data points from the nine systems and then see if any of above strong correlations will survive. Shown in Fig. 3 are the results from the energetic analysis, where correlations of $\Delta \mathrm{E}$ with one and two component fittings are exhibited. As can be seen from Fig. 3a, with all data points put together, $\Delta \mathrm{E}_{\mathrm{e}}$ is still strongly correlated with $\Delta \mathrm{E}$ with the correlation coefficient $\mathrm{R}^{2}=0.981$. This result discloses that, no matter how small or bulky the size of the alkane groups is, the rotation barrier of the $\mathrm{C}-\mathrm{C}$ bond is dominated by the electrostatic interaction. For other components such as $\Delta \mathrm{E}_{\mathrm{xc}}, \Delta \mathrm{E}_{\mathrm{S}}$ and $\Delta \mathrm{E}_{\mathrm{q}}$, even though their correlations with $\Delta \mathrm{E}$ is still 
reasonable and positive, but none of them is as strong as $\Delta \mathrm{E}_{\mathrm{e}}$. However, if they are paired with $\Delta \mathrm{E}_{\mathrm{e}}$ to perform two-variable least-square fittings, as shown in Figs. $3 \mathrm{e}$ and $3 \mathrm{f}$, markedly improved correlations can be obtained. These results are in excellent agreement with our previous work about confirmational stability for other systems,,$^{11,14,16,17,20}$ indicating that it is the electrostatic interaction that plays the dominant role with steric and exchange-correlation effects being minor yet indispensable. These results also suggest that even though those dimers with bulky alkane groups look special, they are indeed no exceptions. At least with the nine models studied in this work, their rotation barriers are the same as simple systems and dominated by the electrostatic interaction.

Figures 4 and 5 are strong correlations of the rotation barrier $\Delta \mathrm{E}$ with informationtheoretic quantities at molecular and atomic levels, respectively. Figure 4a shows the correlation with the Ghosh-Berkowitz-Parr entropy, whose correlation coefficient $\mathrm{R}^{2}$ equal to 0.903. When five ITA quantities are fitted together, as demonstrated previously by us, ${ }^{29-31}$ a much better correlation with $\mathrm{R}^{2}=0.994$, will be obtained (Fig. 4b). At the atomic level, as can be seen from Fig. 5 , strong correlations of $\Delta \mathrm{E}$ for all nine compounds with Fisher information and relative Fisher information of the carbon atoms on the $C-C$ bond with $R^{2}$ better than 0.90 . These atomic results are in contrast with the correlation in Fig. $3 \mathrm{c}$ at the molecular level. From the energetic viewpoint, steric effect, ${ }^{10}$ which is $1 / 8$ of the Fisher information, ${ }^{20}$ is not significantly correlated with $\Delta \mathrm{E}$. However, at the atomic level, for the carbons of the C-C single bond, Fisher information and relative Fisher information ${ }^{21,23}$ are closely related to the rotation barrier height.

With data points from all nine compounds combined, we are still able to observe strong linear correlations of the $\mathrm{C}-\mathrm{C}$ bond rotation barrier height with numerous electronic properties. These strong correlations illustrate that the generation of the $\mathrm{C}-\mathrm{C}$ bond rotation barrier is multifaceted and synergetic, and multiple interactions work together cooperatively to give arise the barrier height. More importantly, for dimeric model systems with bulky alkane 
groups in Scheme 1, no matter how large or small the alkane groups are, they are no exceptions and they obey the same rule with the same patterns discovered. For these systems, dispersion forces might have played a role, but our present results firmly validate that it is the electrostatic interaction that plays the dominant role with the contributions from steric and exchange-correlation effects minor but indispensable.

In summary, using nine dimeric models with bulky alkane groups, in this work, we have examined the nature of the rotation barrier of the carbon-carbon single bond with two density-based total energy decomposition schemes and information-theoretic approach in density functional theory. Exceedingly elongated carbon-carbon bond distances were unveiled. Our results show that the rotation barrier generation is synergetic and multifaceted in nature. One consequence of this study is the insight about the origin of the stability of these bulky dimers. Historically, dispersion forces are believed to be mainly responsible for their stability. Nevertheless, our present results invalidate that argument and confirm that the dominant factor is the electrostatic interaction and contributions from steric and exchangecorrelation effects are minor yet indispensable.

Supporting Information Available: Model building and computational details, together with the Cartesian coordinate of all the optimized structures at the DFT M062X/6-311G(d,p) level of theory from the flexible rotation of nine compounds discussed in this work are available free of charge via the Internet at http://pubs.acs.org.

\section{ACKNOWLEDGMENTS}

We are grateful to Dr. Dongbo Zhao of Yunnan University for helpful discussion. XJW and CYR acknowledge support from the science and technology innovation Program of Hunan Province (2021RC1003). 


\section{References}

1. Schreiner, P. R.; Chernish, L. V.; Gunchenko, P. A.; Tikhonchuk, E. Y.; Hausmann, H.; Serafin, M.; Schlecht, S.; Dahl, J. E. P.; Carlson, R. M. K.; Fokin, A. A. Overcoming lability of extremely long alkane carbon-carbon bonds through dispersion forces. Nature 2011, 477, 308-311.

2. Kammermeier, S.; Herges, R.; Jones, P. G. [2+ 2] Cycloaddition Products of Tetradehydrodianthracene: Experimental and Theoretical Proof of Extraordinary Long CC Single Bonds. Angew. Chem., Int. Ed. Engl. 1997, 36, 1757- 1760.

3. Fokin, A. A.; Chernish, L. V.; Gunchenko, P. A.; Tikhonchuk, E. Y.; Hausmann, H.; Serafin, M.; Dahl, J. E. P.; Carlson, R. M. K.; Schreiner, P. R. Stable Alkanes Containing Very Long Carbon-Carbon Bonds. J. Am. Chem. Soc. 2012, 134, 13641- 13650.

4. Suzuki, T.; Uchimura, Y.; Nagasawa, F.; Takeda, T.; Kawai, H.; Katoono, R.; Fujiwara, K.; Murakoshi, K.; Fukushima, T.; Nagaki, A.; Yoshida, J. I. Expandability of Ultralong C-C Bonds: Largely Different C1-C2 Bond Lengths Determined by Low-Temperature X-ray Structural Analyses on Pseudopolymorphs of 1, 1-bis (4-fluorophenyl)-2, 2-bis (4methoxyphenyl) Pyracene. Chem. Lett. 2014, 43, 86- 88.

5. Ishigaki, Y.; Shimajiri, T.; Takeda, T.; Katoono, R.; Suzuki, T. Longest C-C single bond among neutral hydrocarbons with a bond length beyond $1.8 \AA$ A. Chem. 2018, 4, 795- 806.

6. Himajiri, T.; Suzuki, T.; Ishigaki, Y. Flexible C-C Bonds: Reversible Expansion, Contraction, Formation, and Scission of Extremely Elongated Single Bonds. Angew. Chem., Int. Ed. Engl. 2020, 59, 22252-22257.

7. Takeda, T.; Kawai, H.; Herges, R.; Mucke, E.; Sawai, Y.; Murakoshi, K.; Fujiwara, K.; Suzuki, T. Negligible Diradical Character for the Ultralong C-C Bond in 1, 1, 2, 2Tetraarylpyracene Derivatives at Room Temperature. Tetrahedron Lett. 2009, 50, 36933697.

8. Kuroda, Y.; Kobayashi, M.; Taketrugu, T. Dispersion Interaction and Crystal Packing Realize an Ultralong C-C Single Bond: A Theoretical Study on Dispirobis(10-methylacridan) Derivatives. Chem. Lett. 2019, 48, 137- 139.

9. Yang, J.-X.; Wang, Y.A. How Long Can A C-C $\sigma$-Bond Be? J. Phys. Chem. A 2021, 125, 933 942.

10. Liu, S.B. Steric effect: A Quantitative Description from Density Functional Theory. J. Chem. Phys. 2007, 126, 244103.

11. Liu, S.B. Origin and Nature of Bond Rotation Barriers: A Unified View. J. Phys. Chem. A 2013, 17, 962-965. 
12. Liu, S.B.; Hu, H.; Pedersen, L.G. Steric, Quantum, and Electrostatic Effects on SN2 Reaction Barriers in Gas Phase. J. Phys. Chem. A 2010, 114, 5913-5918.

13. Liu, S.B.; Schauer, C.K. Origin of Molecular Conformational Stability: Perspectives from Molecular Orbital Interactions and Density Functional Reactivity Theory. J. Chem. Phys. 2015, 142, 054107.

14. Liu, S.B. Conceptual Density Functional Theory and Some Recent Developments. Acta Phys.-Chim. Sin. 2009, 25, 590-600.

15. Liu, S.B. Information-Theoretic Approach in Density Functional Reactivity Theory. Acta Phys.-Chim. Sin. 2016, 32, 98- 118.

16. Liu, S.B. Homochirality Originates from Handedness of Helices. J. Phys. Chem. Lett. 2020, $11,8690-8696$.

17. Li, M.; He, X.; Chen, J.; Wang, B.; Liu, S.B.; Rong, C. Density Functional Theory and Information-Theoretic Approach Study on the Origin of Homochirality in Helix Structures. J. Phys. Chem. A 2021, 125, $1269-1278$.

18. Liu, S.B. Principle of Chirality Hierarchy in Three-Blade Propeller Systems. J. Phys. Chem. Lett. 2021, 12, 8720-8725.

19. Glendening, E.D.; Landis, C.R.; Weinhold, F. NBO 7.0: New Vistas in Localized and Delocalized Chemical Bonding Theory. J. Comput. Chem. 2019, 40, 2234 - 2241.

20. Rong, C.; Wang, B.; Zhao, D.; Liu, S.B. Information-Theoretic Approach in Density Functional Theory and its Recent Applications to Chemical Problems. Wiley Interdiscip. Rev.: Comput. Mol. Sci. 2020, 10, e1461.

21. Liu, S.B. On the Relationship between Densities of Shannon Entropy and Fisher Information for Atoms and Molecules. J. Chem. Phys. 2007, 126, 191107.

22. Ghosh, S. K.; Berkowitz, M.; Parr, R. G. Transcription of Ground-State Density-Functional Theory into a Local Thermodynamics. Proc. Natl. Acad. Sci. U.S.A. 1984, 81, 8028-8031.

23. Liu, S.B. Identity for Kullback-Leibler Divergence in Density Functional Reactivity Theory. J. Chem. Phys. 2019, 151, 141103.

24. Wang, B.; Zhao, D.; Lu, T.; Liu, S.B.; Rong, C. Quantifications and Applications of Relative Fisher Information in Density Functional Theory. J. Phys. Chem. A 2021, 125, 3802- 3811.

25. Liu, S. B.; Rong, C.; Lu, T. Information Conservation Principle Determines Electrophilicity, Nucleophilicity, and Regioselectivity. J. Phys. Chem. A 2014, 118, 3698- 3704.

26. Liu, S. B. Quantifying Reactivity for Electrophilic Aromatic Substitution Reactions with Hirshfeld Charge. J. Phys. Chem. A 2015, 119, 3107- 3111. 
27. Guo, C.; He, X.; Rong, C.; Lu, T.; Liu, S.B.; Chattaraj, P.K. Local Temperature as a Chemical Reactivity Descriptor. J. Phys. Chem. Lett. 2021, 12, 23, 5623 - 5630.

28. Grimme, S.; Antony, J.; Ehrlich, S.; Krieg, H. A Consistent and Accurate Ab Initio Parameterization of Density Functional Dispersion Correction (DFT-D) for the 94 Elements H-Pu. J. Chem. Phys. 2010, 132, 154104.

29. Zhou, X.; Rong, C.; Lu, T.; Zhou, P.; Liu, S. B. Information Functional Theory: Electronic Properties as Functionals of Information for Atoms and Molecules. J. Phys. Chem. A 2016, $120,3634-3642$.

30. Cao, X.; Rong, C.; Zhong, A.; Lu, T.; Liu, S.B. Molecular Acidity: An Accurate Description with Information-Theoretic Approach in Density Functional Theory. J. Comp. Chem. 2018, 39, $117-129$.

31. Xiao, X.; Cao, X.; Zhao, D.; Rong, C.; Liu, S.B. Quantification of Molecular Basicity for Amines: A Combined Conceptual Density Functional Theory and Information-Theoretic Approach Study. Acta. Phys.-Chim. Sin. 2020, 36, 1906034. 
Table 1. Correlation coefficient ( $\mathrm{R}$ ) values of the total energy difference $\Delta \mathrm{E}$ (with the conformation of $\angle \mathrm{C}-\mathrm{C}-\mathrm{C}-\mathrm{C}=0^{\circ}$ as the reference) for each of the nine systems studied in this work with five energy components, kinetic energy $\Delta T_{S}$, exchange-correlation energy $\Delta E_{x c}$, electrostatic energy $\Delta \mathrm{E}_{\mathrm{e}}$, steric energy $\Delta \mathrm{E}_{\mathrm{s}}$, and Fermionic quantum energy $\Delta \mathrm{E}_{\mathrm{q}}$, four information-theoretic quantities, Fisher information $\Delta \mathrm{I}_{\mathrm{F}}$, Ghosh-Berkowitz-Parr entropy $\Delta \mathrm{S}_{\mathrm{GBP}}$, relative Fisher information $\Delta \mathrm{G}_{3}$ and alterative relative Fisher information $\Delta \mathrm{G}_{1}$, plus five other electronic properties including the HOMO/LUMO gap $(\triangle \mathrm{GAP})$, valence ( $\triangle$ Valence) and Rydberg ( $\Delta$ Rydberg) contributions of non-Lewis orbitals, the occupancy of the C-C bond ( $\triangle$ Ocсpcy) and total hyperconjugation interactions of the entire system ( $\Delta$ Hyprcnj) from the NBO analysis.

\begin{tabular}{lccccccccc}
\hline Property & $\mathbf{1}$ & $\mathbf{2}$ & $\mathbf{3}$ & $\mathbf{4}$ & $\mathbf{5}$ & $\mathbf{6}$ & $\mathbf{7}$ & $\mathbf{8}$ & $\mathbf{9}$ \\
\hline$\Delta \mathrm{T}_{\mathrm{S}}$ & -0.988 & -0.996 & -0.969 & -0.993 & -0.993 & -0.991 & -0.969 & -0.994 & -0.993 \\
$\Delta \mathrm{E}_{\mathrm{xc}}$ & 0.991 & 0.999 & 0.989 & 0.986 & 0.913 & 0.914 & 0.987 & 0.990 & 0.946 \\
$\Delta \mathrm{E}_{\mathrm{e}}$ & 0.996 & 0.999 & 0.995 & 0.999 & 0.993 & 0.991 & 0.987 & 0.996 & 0.993 \\
$\Delta \mathrm{E}_{\mathrm{s}}$ & 0.996 & 0.999 & 0.993 & 0.978 & 0.834 & 0.808 & 0.983 & 0.981 & 0.906 \\
$\Delta \mathrm{E}_{\mathrm{q}}$ & 0.997 & 0.999 & 0.990 & 0.974 & 0.709 & 0.573 & 0.961 & 0.964 & 0.802 \\
$\Delta \mathrm{I}_{\mathrm{F}}$ & 0.999 & 0.999 & 0.993 & 0.979 & 0.833 & 0.885 & 0.983 & 0.981 & 0.907 \\
$\Delta \mathrm{G}_{1}$ & -0.987 & -0.999 & -0.976 & -0.996 & -0.914 & -0.847 & -0.879 & -0.916 & -0.915 \\
$\Delta \mathrm{G}_{3}$ & -0.904 & -0.937 & -0.950 & -0.857 & -0.599 & -0.64 & -0.911 & -0.968 & -0.724 \\
$\Delta \mathrm{S}_{\mathrm{GBP}}$ & 0.999 & 0.996 & 0.956 & 1.000 & 0.746 & 0.899 & 0.961 & 0.976 & 0.871 \\
& & & & & & & & & \\
$\Delta \mathrm{GAP}$ & -0.757 & -0.964 & -0.393 & -0.934 & -0.056 & -0.365 & -0.905 & -0.667 & -0.639 \\
$\Delta$ Valence & -0.992 & -1.000 & -0.935 & -0.943 & 0.285 & 0.481 & 0.851 & 0.910 & 0.762 \\
$\Delta$ Rydberg & 0.953 & 0.986 & 0.529 & 0.849 & 0.108 & 0.948 & 0.548 & 0.464 & 0.732 \\
$\Delta$ Occpcy & 0.990 & 0.999 & 0.277 & 0.980 & 0.430 & -0.288 & 0.975 & 0.813 & -0.429 \\
$\Delta$ Hyprcnj & -0.939 & -0.910 & -0.705 & -0.966 & -0.483 & 0.690 & 0.710 & 0.405 & 0.247 \\
\hline
\end{tabular}


Table 2. Correlation coefficient $(\mathrm{R})$ values of the total energy difference $\Delta \mathrm{E}$ (with the conformation of $\angle \mathrm{C}-\mathrm{C}-\mathrm{C}-\mathrm{C}=0^{\circ}$ as the reference) at the atomic and bond levels for each of the nine systems studied in this work. Quantities considered include the $\mathrm{C}-\mathrm{C}$ bond distance $\mathrm{R}_{\mathrm{c}-\mathrm{c}}$, Hirshfeld charges on the two carbon atoms of the $\mathrm{C}-\mathrm{C}$ bond, density-based local temperature on the carbon atoms, and atomic contributions of information gain $\Delta \mathrm{I}_{\mathrm{G}}$, Shannon entropy $\Delta \mathrm{S}_{\mathrm{S}}$, Fisher information $\Delta \mathrm{I}_{\mathrm{F}}$, two relative Fisher information $\Delta \mathrm{G}_{1}$ and $\Delta \mathrm{G}_{3}$, second-order Rényi entropy $\Delta R_{2}$ and second-order relative Rényi entropy $\Delta^{r} R_{2}$. The Hirshfeld partition of atoms in molecules was employed.

\begin{tabular}{lrrrrrrrrr}
\hline Property & 1 & 2 & 3 & 4 & 5 & 6 & 7 & 8 & 9 \\
\hline $\mathrm{R}_{\mathrm{C}-\mathrm{C}}$ & 0.997 & 1.000 & 0.984 & 0.998 & 0.753 & 0.478 & 0.597 & 0.767 & 0.777 \\
CHirsheld & 0.976 & 0.989 & 0.950 & 0.977 & 0.724 & 0.850 & 0.989 & 0.990 & 0.227 \\
CHirsheld & 0.976 & 0.989 & 0.950 & 0.977 & 0.724 & 0.841 & 0.985 & 0.994 & 0.598 \\
& & & & & & & & & \\
LocalTC1 $_{\mathrm{C}}$ & 0.992 & 0.998 & 0.957 & 0.995 & 0.958 & 0.891 & 0.982 & 0.967 & 0.924 \\
LocalTC2 $_{\mathrm{C}}$ & 0.992 & 0.998 & 0.909 & 0.995 & 0.932 & 0.824 & 0.990 & 0.975 & 0.926 \\
$\Delta \mathrm{I}_{\mathrm{G}}$ & -0.979 & -0.977 & -0.947 & -0.987 & -0.652 & -0.709 & -0.996 & -0.988 & -0.811 \\
$\Delta \mathrm{S}_{\mathrm{S}}$ & -0.986 & -1.000 & -0.945 & -0.974 & 0.736 & 0.675 & -0.252 & 0.740 & 0.835 \\
$\Delta \mathrm{I}_{\mathrm{F}}$ & 0.998 & 0.993 & 0.953 & 0.997 & 0.930 & 0.911 & 0.991 & 0.982 & 0.932 \\
$\Delta \mathrm{G}_{1}$ & -0.985 & -0.948 & -0.905 & -0.981 & -0.857 & -0.827 & -0.971 & -0.975 & -0.780 \\
$\Delta \mathrm{G}_{3}$ & -0.999 & -0.999 & -0.952 & -0.999 & -0.888 & -0.896 & -0.975 & -0.971 & -0.899 \\
$\Delta \mathrm{R}_{2}$ & -0.982 & -0.748 & -0.901 & -0.990 & -0.838 & -0.615 & -0.986 & -0.973 & -0.914 \\
$\Delta^{\mathrm{r}} \mathrm{R}_{2}$ & -0.976 & -0.977 & -0.953 & -0.984 & -0.647 & -0.689 & -0.997 & -0.991 & -0.729 \\
\hline
\end{tabular}


Table 3. Benchmark results of basis sets and methods for Compound 5 in Scheme 1. Six different basis sets and six methods have been chosen. $\mathrm{R}$ stands for the optimization $\mathrm{C}-\mathrm{C}$ bond distance. Two correlation coefficients of $\Delta \mathrm{E}$ with $\Delta \mathrm{E}_{\mathrm{xc}}$ and $\Delta \mathrm{E}_{\mathrm{e}}$ are tabulated in the benchmark test. CBSB7 and MP2 could be regarded as the reference in these tests. DC stands for dispersion correction. The experimental C-C bond distance value of 5 is 1.647A. In basis set tests, M062X functional was employed and in methodology tests, 6-311G(d,p) basis set was utilized.

\begin{tabular}{lccc|lccc}
\hline Basis set & $\mathrm{R}$ & $\Delta \mathrm{E}_{\mathrm{xc}}$ & $\Delta \mathrm{E}_{\mathrm{e}}$ & Method & $\mathrm{R}$ & $\Delta \mathrm{E}_{\mathrm{xc}}$ & $\Delta \mathrm{E}_{\mathrm{e}}$ \\
\hline 6-31G(d) & 1.648 & 0.924 & 0.996 & B3LYP & 1.673 & 0.978 & 0.996 \\
6-311G(d,p) & 1.649 & 0.913 & 0.993 & B3LYP+DC & 1.653 & 0.972 & 0.996 \\
6-311++G(d,p) & 1.649 & 0.917 & 0.993 & M062X & 1.649 & 0.911 & 0.992 \\
DGDZVP & 1.648 & 0.925 & 0.988 & TPSSTPSS & 1.668 & 0.987 & 0.988 \\
cC-pVTZ & 1.647 & 0.920 & 0.993 & WB97XD & 1.649 & 0.965 & 0.981 \\
CBSB7 & 1.649 & 0.913 & 0.993 & MP2 & 1.640 & 0.968 & 0.998 \\
\hline
\end{tabular}




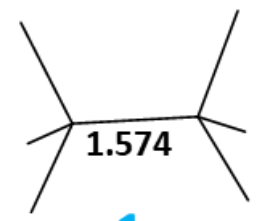

1

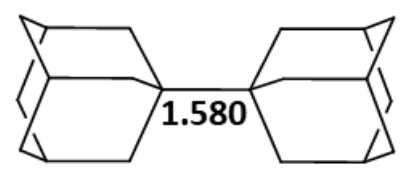

4

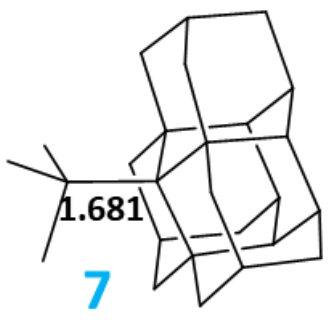

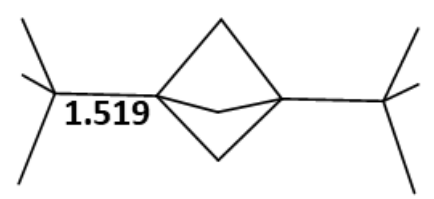

2

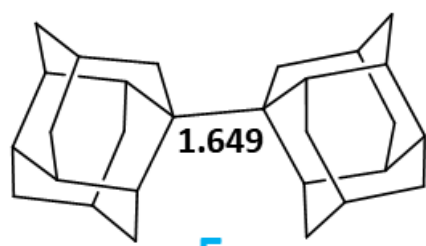

5

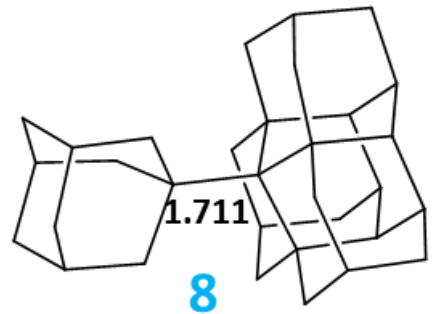

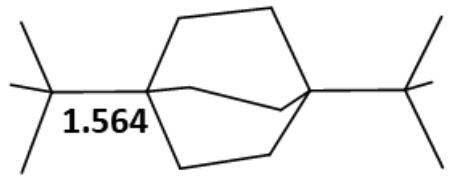

3
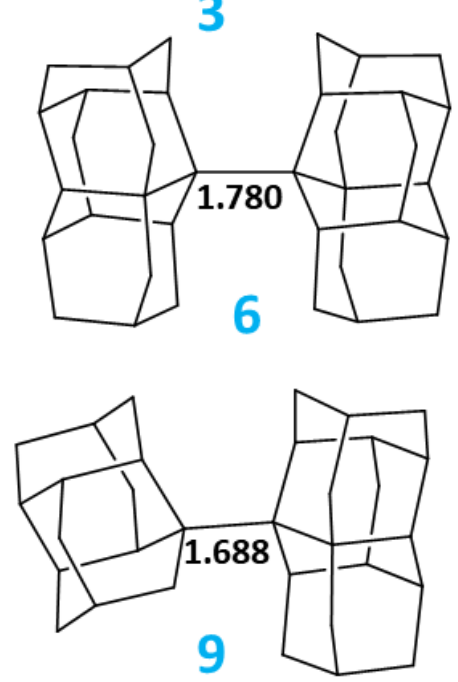

Scheme 1. Nine alkane dimers studied in this work. Terminal bulky alkane groups include tertbutyl, diamantane, triamantane and tetramantane. The optimized C-C bond distance for each compound at the DFT M062X/6-311G(d,p) level of theory is also shown. Flexible rotation was conducted from $0^{\circ}$ to $360^{\circ}$ with the step size of $5^{\circ}$ for the C-C single bond connected with the two bulky alkane groups, except for $\mathbf{2}$ and 3, where bicyclo[1,1,1]pentane in $\mathbf{2}$ and bicyclo[2,2,2]octane in $\mathbf{3}$ were held fixed and two terminal terbutyl groups were rotated. 


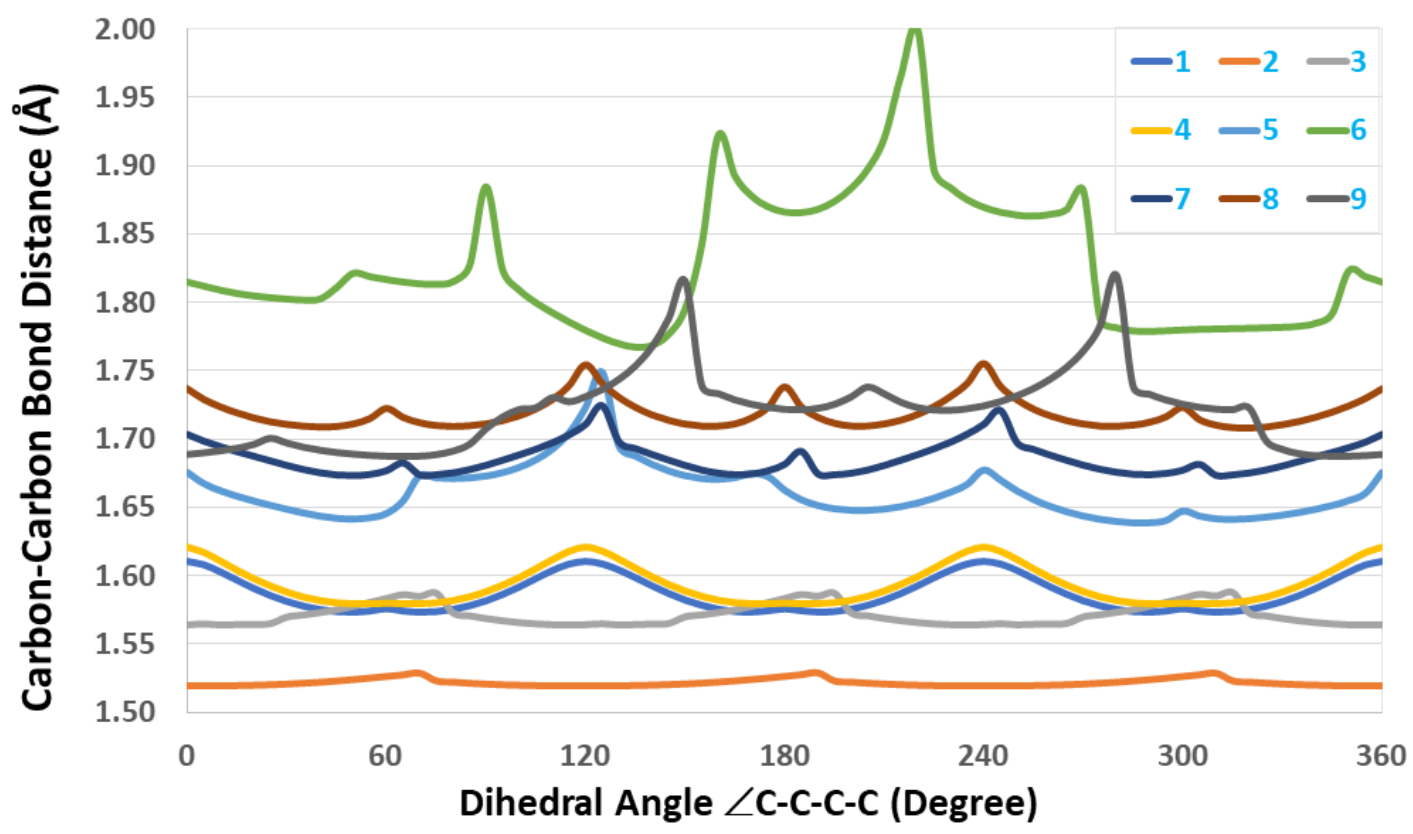

Figure 1. The profile of carbon-carbon bond distance as a function of the flexible C-C single bond rotation from $0^{\circ}$ to $360^{\circ}$ for nine compounds in Scheme 1. 


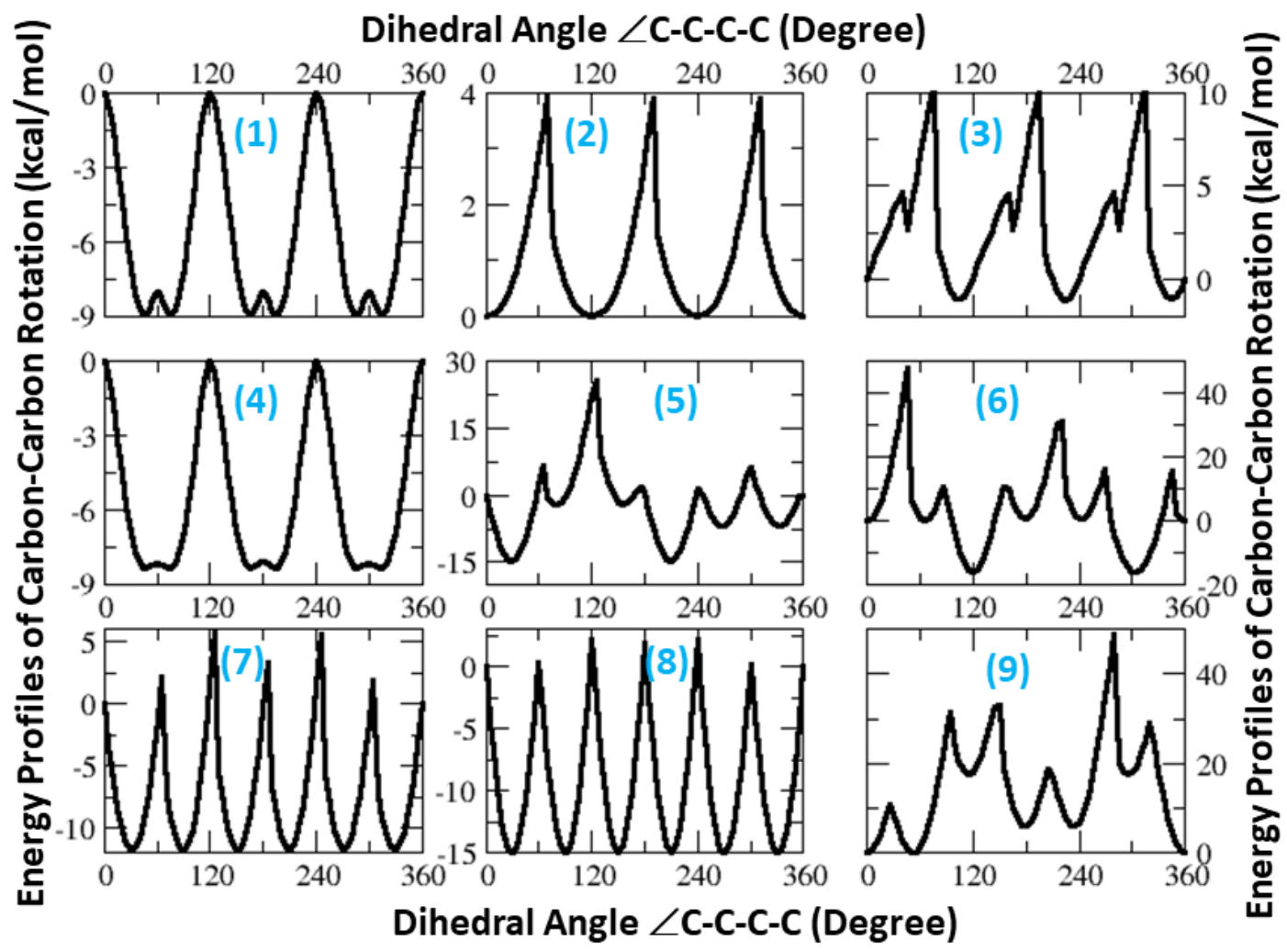

Figure 2. The total energy profile of nine compounds in Scheme 1 as a function of $\mathrm{C}-\mathrm{C}$ bond rotation from $0^{\circ}$ to $360^{\circ}$ using the conformation with the dihedral angle $\angle \mathrm{C}-\mathrm{C}-\mathrm{C}-\mathrm{C}=0^{\circ}$ as the reference point. 

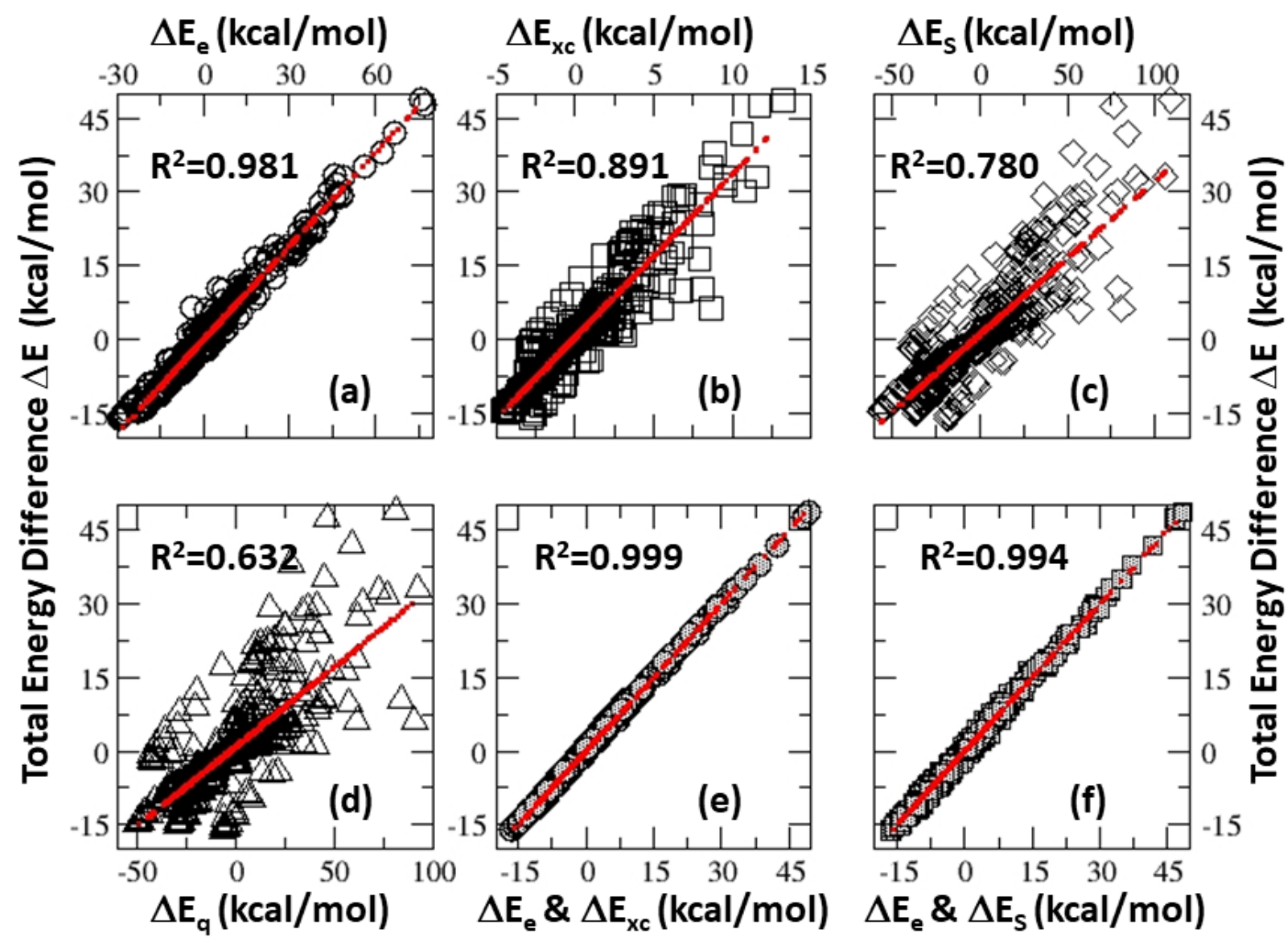

Figure 3. Positive correlations of the total energy difference $\Delta \mathrm{E}$ for all nine systems in Scheme 1 (with the dihedral angle $\angle \mathrm{C}-\mathrm{C}-\mathrm{C}-\mathrm{C}=0^{\circ}$ as the reference point) with different energy components from the two total energy partition schemes in DFT. Plots (a)-(d) are single component least-square fittings with (a) electrostatic $\Delta \mathrm{E}_{\mathrm{e}}$, (b) exchange-correlation $\Delta \mathrm{E}_{\mathrm{xc}}$, (c) steric $\Delta \mathrm{E}_{\mathrm{s}}$, and (d) Fermionic quantum $\Delta \mathrm{E}_{\mathrm{q}}$ components. Plots (e) \& (f) are two-component fittings with (e) $\Delta \mathrm{E}_{\mathrm{e}} \& \Delta \mathrm{E}_{\mathrm{xc}}$ and (f) $\Delta \mathrm{E}_{\mathrm{e}} \& \Delta \mathrm{E}_{\mathrm{s}}$ components. 


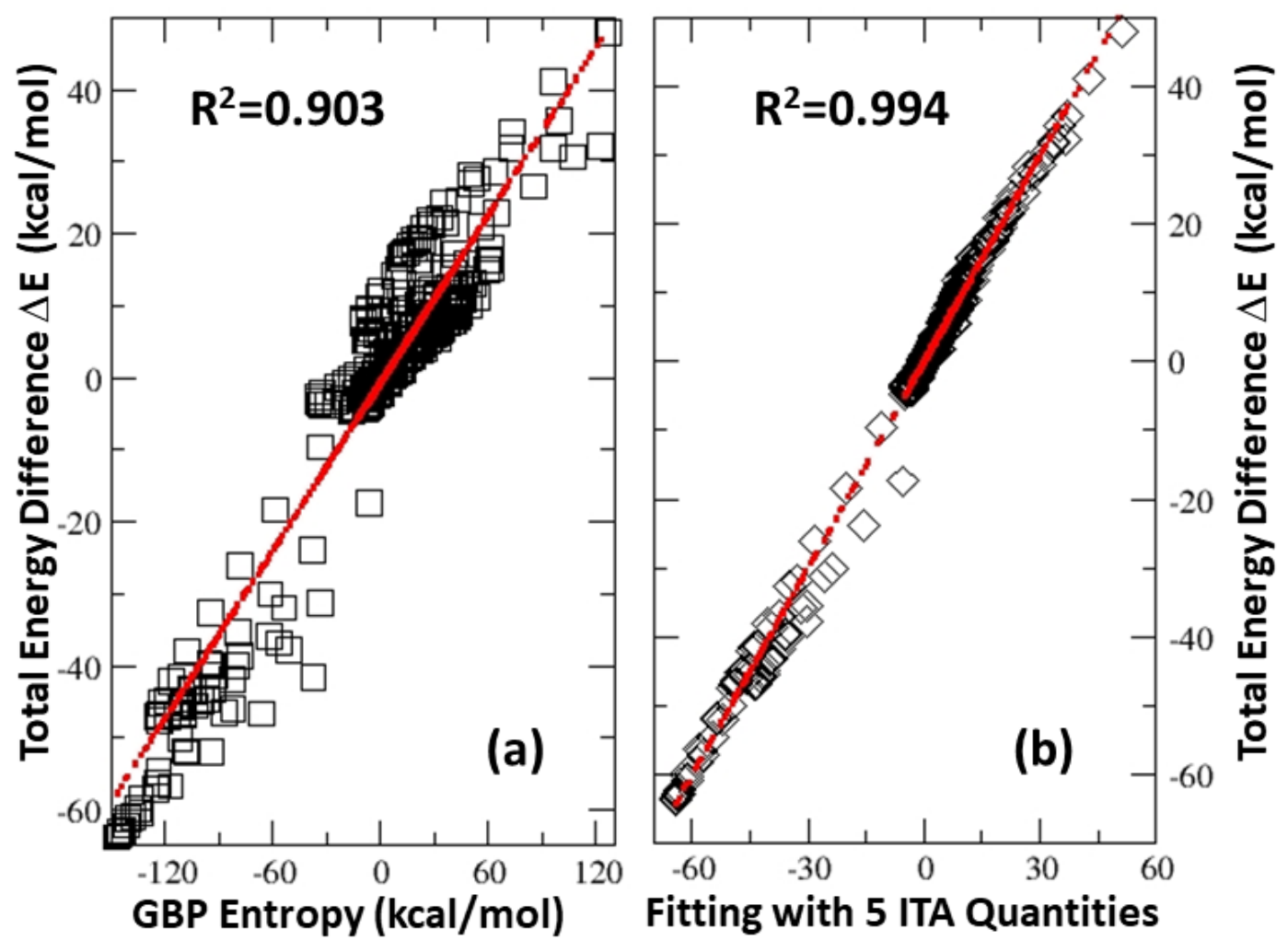

Figure 4. Strong correlations of the total energy difference $\Delta E$ for all nine systems in Scheme 1 (with the dihedral angle $\angle \mathrm{C}-\mathrm{C}-\mathrm{C}-\mathrm{C}=0^{\circ}$ as the reference point) with information-theoretic quantities at the molecular level: (a) the Ghost-Berkowitz-Parr entropy and (b) when fitted with 5 ITA quantities including the Ghost-Berkowitz-Parr entropy, Shannon entropy, relative Shannon entropy, Fisher information and relative Fisher information. 


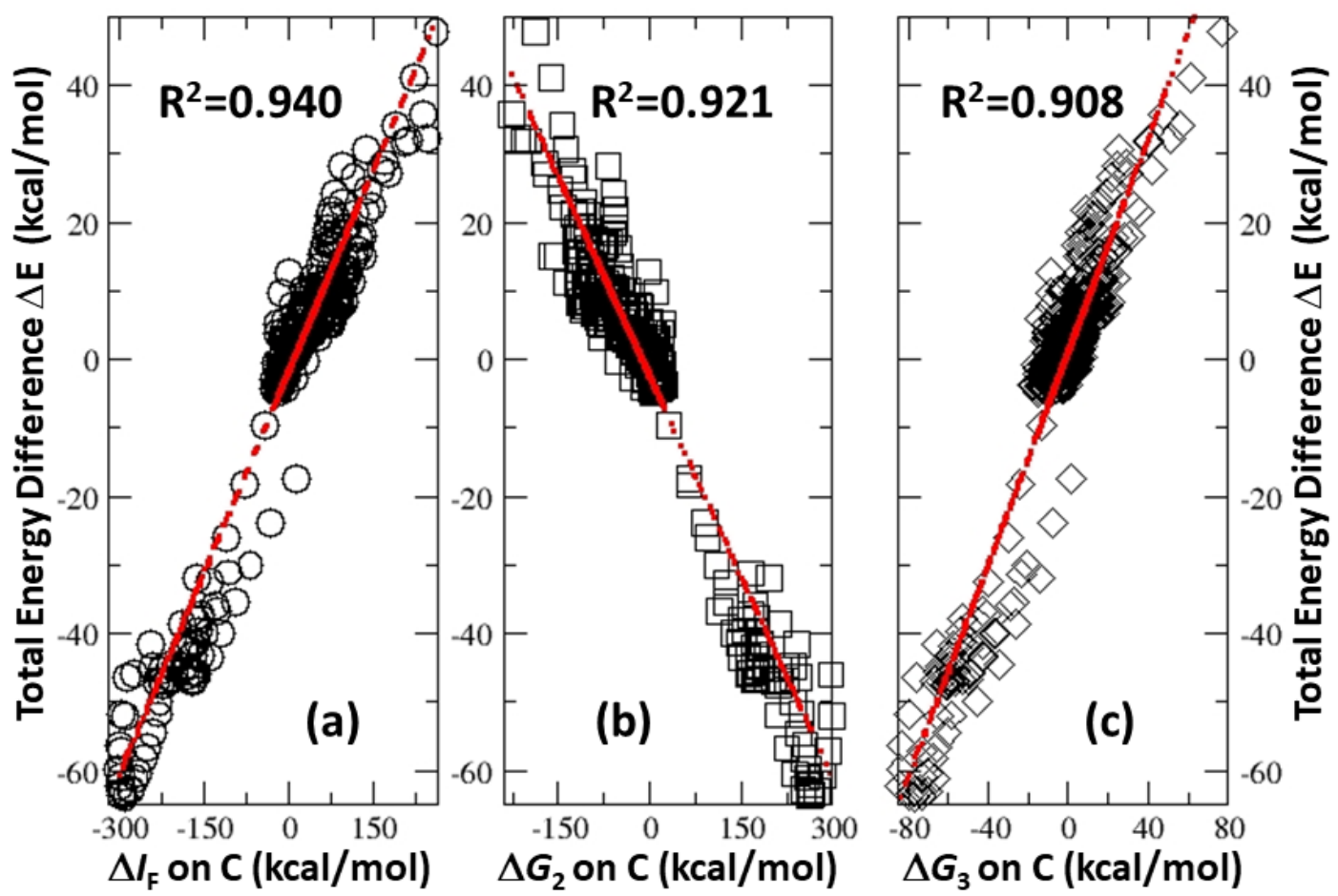

Figure 5. Strong correlations of the total energy difference $\Delta \mathrm{E}$ for all nine systems in Scheme 1 (with the dihedral angle $\angle \mathrm{C}-\mathrm{C}-\mathrm{C}-\mathrm{C}=0^{\circ}$ as the reference point) with information-theoretic quantities at the atomic level: (a) Fisher information $I_{F}$, (b) $G_{2}$ function and (3) $G_{3}$ function (relative Fisher information) on the carbon atom of the $\mathrm{C}-\mathrm{C}$ single bond. 


\section{TOC Graphic}

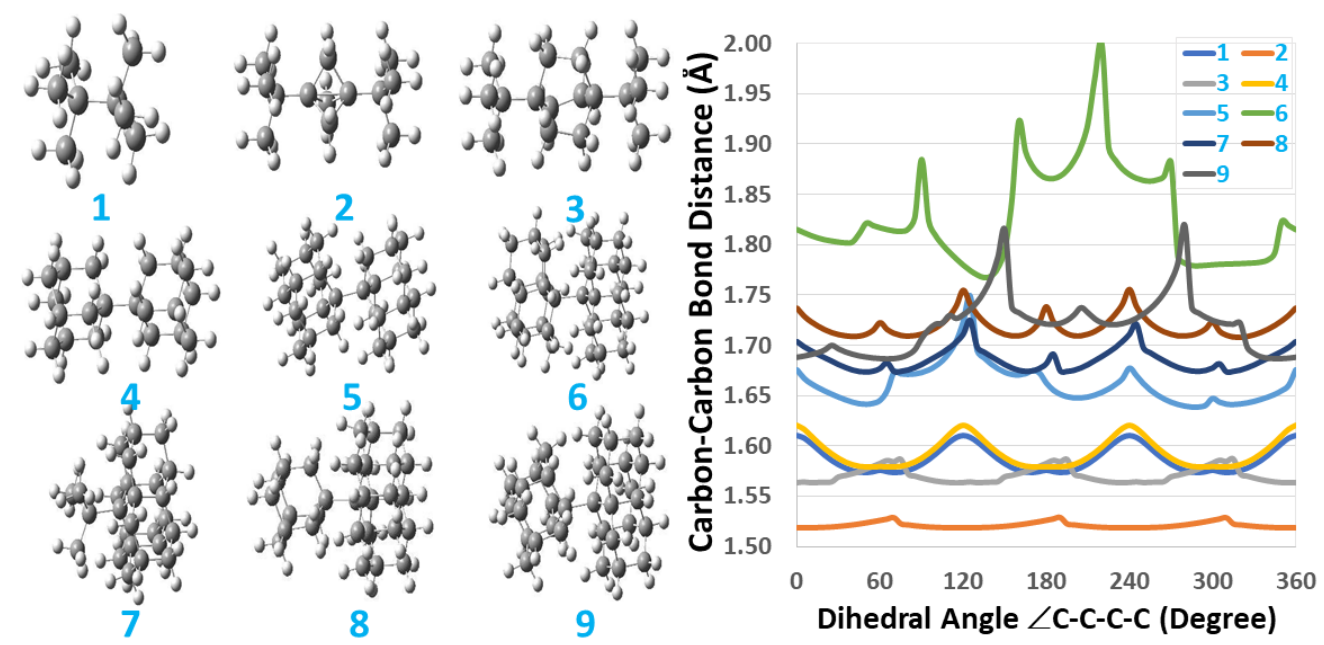

